\title{
Neurotypic Cell Attachment and Growth on III- Nitride Lateral Polarity Structures
}

\author{
L. E. Bain ${ }^{\mathrm{a}}$, R. Kirste ${ }^{\mathrm{b}}$, C.A. Johnson ${ }^{\mathrm{c}}$, H.T. Ghashghaei ${ }^{\mathrm{c}}$, R. Collazo ${ }^{\mathrm{b}}$, and A. \\ Ivanisevic ${ }^{b^{*}}$
}

*Corresponding Author, email: Ivanisevic@ncsu.edu

\begin{abstract}
III-nitride materials have recently received increasing levels of attention for their potential to successfully interface with, and sense biochemical interactions in biological systems. Expanding on available sensing schemes (including transistor-based devices,) a III-N lateral polarity structure capable of introducing quasi phase matching through a periodic polarity grating presents a novel platform for second harmonic generation. This platform constitutes a nonlinear optical phenomenon with exquisite sensitivity to the chemical state of a surface or interface. To characterize the response of a biological system to the nanostructured lateral polarity structures, we cultured neurotypic PC12 cells on AlGaN with varying ratios of Al:Ga - 0, 0.4, 0.6, and 1 - and on surfaces of varying pitch to the III-polar vs. N-polar grating - 5, 10, 20 and $50 \mu \mathrm{m}$. While some toxicity associated with increasing Al is observed, we documented and quantified trends in cell responses to the local material polarity and nanoscale roughness. The nitrogenpolar material has a significantly higher nanoscale roughness than III-polar regions, and a 80-200 nm stepheight difference between the III-polar and N-polar material in the lateral polarity configuration generates adequate changes in topography to influence cell growth, improves cell adhesion and promotes cell migration along the direction of the features. As the designed material configuration is further explored for biochemical sensing, the lateral polarity scheme may provide a route in assessing the non-specific protein adsorption to this varying nano-topography that drives the subsequent cell response.
\end{abstract}

\section{Introduction}

Recent interest in developing medical technologies and devices based on functional material integration has led to the investigation of semiconductors for interfacing with biological entities. ${ }^{1}$ Chemical stability ${ }^{2}$, biocompatibility, ${ }^{3-5}$ and multiple routes of functionality (surface-sensitive field effect transistors (FETs), ${ }^{6}$ light emitting diodes (LEDs), ${ }^{7-9}$ or schemes involving nonlinear optical phenomena - including second harmonic generation $(\mathrm{SHG})^{10}$ ) have repeatedly indicated that III-nitride materials are of particular interest. Recent fabrication of an $\mathrm{Al}_{\mathrm{x}} \mathrm{Ga}_{1-\mathrm{x}} \mathrm{N}$ lateral polarity structure (LPS) presents a novel platform for accessing a range of these associated phenomena, including fabrication of LEDs at inversion domain boundaries (the transition from III-polar to N-polar material) or quasiphase matching for SHG. Prior to fabrication of large-scale periodic lateral polarity structures, initial studies of lateral polarity junctions in GaN demonstrated a key characteristic of these features: the potential to form a p-n junction at the interface between the Ga-polar and N-polar material. ${ }^{11}{ }^{12}$ This potential to create functional devices (including LEDs or FETs) through lateral growth of a III-N material was further expanded by demonstration of laterally defined growth of AlN with IIIand N-polar regions. ${ }^{13,14}$ Through integration of both $\mathrm{Al}$ and
Ga, a new method by which material characteristics can be tuned was introduced, allowing greater flexibility in the bandgap and other device characteristics ${ }^{15}$. Coupled with the ability to generate $\mathrm{p}-\mathrm{n}$ junctions during material growth, the $\mathrm{Al}_{\mathrm{x}} \mathrm{Ga}_{1-\mathrm{x}} \mathrm{N}$ LPS thus presents a highly compelling platform for integrated device construction with highly tunable characteristics.

Investigating the response of cells - neurotypic PC12 cells, specifically - to the LPS features has parallels to other investigations of interactions taking place at cell-substrate interfaces. ${ }^{16}$ Contact guidance along grooved surfaces is a well-established phenomenon wherein cell structures align with an underlying physical grating. ${ }^{17-19}$ While containing an analogous micron-scale grating, rather than physical grooves of material (as in studies of contact guidance along grooved polystyrene surfaces) a more nuanced change in the surface condition is produced by the alternating material polarity. In addition to presenting a different compositional face (N-polar vs. Al/Ga-polar,) a concomitant change in nanoscale surface roughness provides a compositionally and topographically patterned surface ${ }^{20}$. This roughness variation is twofold; in addition to the $\mathrm{N}$-polar regions having significantly higher nanoscale surface roughness than $\mathrm{Al} / \mathrm{Ga}$-polar, the structures used in this work have a height difference between the $\mathrm{N}$-polar 
and Al/Ga-polar regions, producing a micron-scale ridge pattern of lateral dimensions corresponding to the fabricated LPS structure. Given prior reports of cells cultured on surfaces with a hierarchical nano-/micro-scale surface texture, ${ }^{17}$ observing any preferential growth on the N-polar vs. Al/Gapolar regions as well as any overarching trends with respect to the local LPS width provides insight into mechanisms through which material composition and nanoscale structure can be tuned to optimize cell-surface interactions.

An additional measure of interest is the role of increasing $\mathrm{Al}$ :Ga in the ternary $\mathrm{Al}_{\mathrm{x}} \mathrm{Ga}_{1-\mathrm{x}} \mathrm{N}$ system. While integrating $\mathrm{Al}$ offers improvements to bandgap tunability, providing accessibility to a larger portion of the UV spectrum, AIN has established cytotoxicity. ${ }^{5,16}$ Balancing the potential favourable device characteristics introduced by introduction of $\mathrm{Al}$ with the potential hazard to cell survival will require a solid understanding of the effect of $\mathrm{Al}$ integration on both initial cell attachment as well as longer-term viability. As such, by characterizing the cellular response to both the micro- and nanoscale changes in topography associated with the LPS as well as a change in $\mathrm{Al}: \mathrm{Ga}$, we can better understand biological interactions taking place on these surfaces and consider how to best optimize semiconductor material surfaces for integration in biochemical detection schemes.

\section{Materials and Methods}

\section{Lateral Polarity Structures}

Four wafers of varying $\mathrm{Al}: \mathrm{Ga}$ compositions - 0\% Al, $40 \%$ $\mathrm{Al}, 70 \% \mathrm{Al}$, and $100 \% \mathrm{Al}$ - were fabricated with the lateral polarity structure patterns. For the remaining discussion, wafers will be described as GaN, A140, A170, and AlN, respectively. Lateral polarity structures were grown using methods detailed previously ${ }^{20,21}$; in brief, a lithographically patterned $20 \mathrm{~nm}$ AlN buffer layer on sapphire provided the template for subsequent LPS growth. Patterning produced specific regions of 5, 10, 20, and $50 \mu \mathrm{m}$ wide LPS. Alternating material polarity is produced by this patterned template growth; regions in which the AlN buffer layer is intact will yield $\mathrm{Al} / \mathrm{Ga}$ polar growth, while growth on the bare c-sapphire produces $\mathrm{N}$ polar material. This growth produces sharp interfaces between III-polar and N-polar regions.

\section{Cell Culture}

PC12 cells were grown in Dulbecco's Modified Eagle Medium (DMEM,) high glucose formulation, with $12.5 \%$ horse serum, $2.5 \%$ fetal bovine serum, and $1 \%$ penicillinstreptomycin. Conventional incubation conditions $-37^{\circ} \mathrm{C}$ and $5 \% \mathrm{CO}_{2}$ in a humidified incubator or chamber - were maintained for both cell growth and time lapse imaging. After seeding on LPS wafers at a $5 \times 10^{5}$ cell $/ \mathrm{mL}$ density, $5 \mathrm{~mL}$ per $60 \mathrm{~mm}$ plate, cells were allowed to adhere for 18 hours prior to exposure to nerve growth factor (NGF.) NGF media consisted of DMEM with $1 \%$ horse serum and $50 \mathrm{ng} / \mathrm{mL}$ NGF. NGF media was replaced at day 3 of culture, after culmination of time-lapse imaging, for subsequent observation of cell densities and characteristics at days 3 and 7 of culture. For culture on poly-D-lysine coated sapphire, the poly-D-lysine surface treatment protocol from Sigma Aldrich was used.

\section{Image Acquisition}

Time-lapse imaging was performed on a Nikon $\mathrm{C} 1$ confocal microscope for 48 hours following introduction of NGF media. Cells were kept in a humidified chamber at $5 \% \mathrm{CO}_{2}$ and $37^{\circ} \mathrm{C}$ for the duration of imaging. Day 3 and 7 images were collected using a Leica DMIL inverted microscope with a QImaging Micropublisher 3.3 camera. Contrast adjustment, addition of false colour (where applicable), and other image analysis were performed using ImageJ.

\section{Cell Adhesion Analysis}

Cells were manually counted, with the average adherent cell density for a given LPS width and Al:Ga combination emerging from the averaged counts of multiple regions. In the instance of large, opaque clusters of cells, an estimate of the number of constituents was made based on the average size of individual cells. Values are displayed as an average plus or minus one standard deviation (SD); the SD is calculated based on counts from a minimum of five $500 \times 500 \mu \mathrm{m}^{2}$ regions with a given composition and lateral pitch combination.

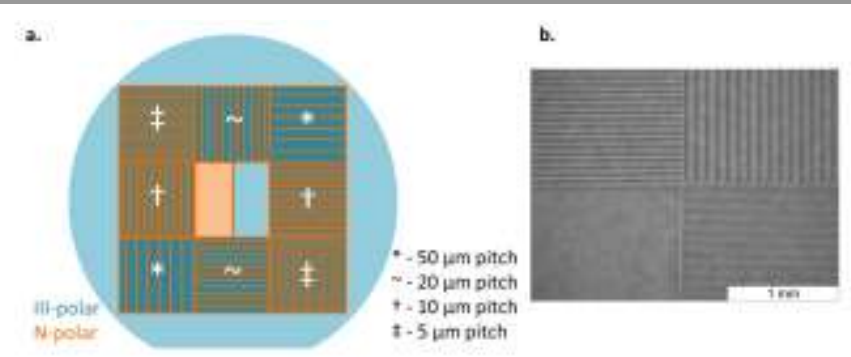

Figure 1 (a) Schematic and (b) optical micrograph of an AlxGa1-xN LPS wafer. III-polar vs. N-polar regions are represented by color, and symbols designate the local LPS width. (b) Optical micrograph of the junction between the III-polar, $10 \mu \mathrm{m}, 20 \mu \mathrm{m}$, and $50 \mu \mathrm{m}$ regions.

\section{Results and Discussion}

The lateral polarity structures were grown on and kept as intact wafers for experiments; this provided a unified platform for simultaneous observation of cell behaviours at different LPS widths. A schematic and optical micrograph of one 'junction' between LPS sections is provided in Figure 1. Each Al:Ga wafer (GaN, A140, A170, AlN) has this overall LPS structure, with one central III-polar region, one central N-polar region, and two regions of each LPS pitch. The III-polar and N-polar central regions provide points of comparison for cell growth on surfaces without the periodic alternating polarization; given the increased roughness of the $\mathrm{N}$-polar material, ${ }^{20}$ this also serves as a metric for the influence of this polarization-associated topographic change on cell adhesion.

The primary mode of analysis in this study involves adherent cell density, a parameter associated with healthy cell- 
substrate connectivity, in a manner involving both initial cell attachment and subsequent viability and integrity of cell adhesion over time. A summary of the final (day 7) adherent cell density is provided in Figure 2. We noted a stark difference in adherent cell density on GaN vs. the Al-containing wafers; by day 7 , cell populations were significantly reduced on Al-containing samples (adherent cell density dropped by $50 \%$ between day 3 and day 7 on both the $40 \%$ and $70 \% \mathrm{Al}$ samples.) The substantial loss in adherent, viable cells on the $70 \% \mathrm{Al}$ sample contributes to the observed high variation in the number of adherent cells; of the five regions identified randomly and imaged for analysis, only a fraction contained adherent cells. The adherent cell population is also substantially reduced on the $40 \% \mathrm{Al}$ sample when compared with the GaN sample. It is for this reason that we limit our subsequent analysis and discussion of preferential cellular attachment and growth with respect to the LPS to the GaN material surface.

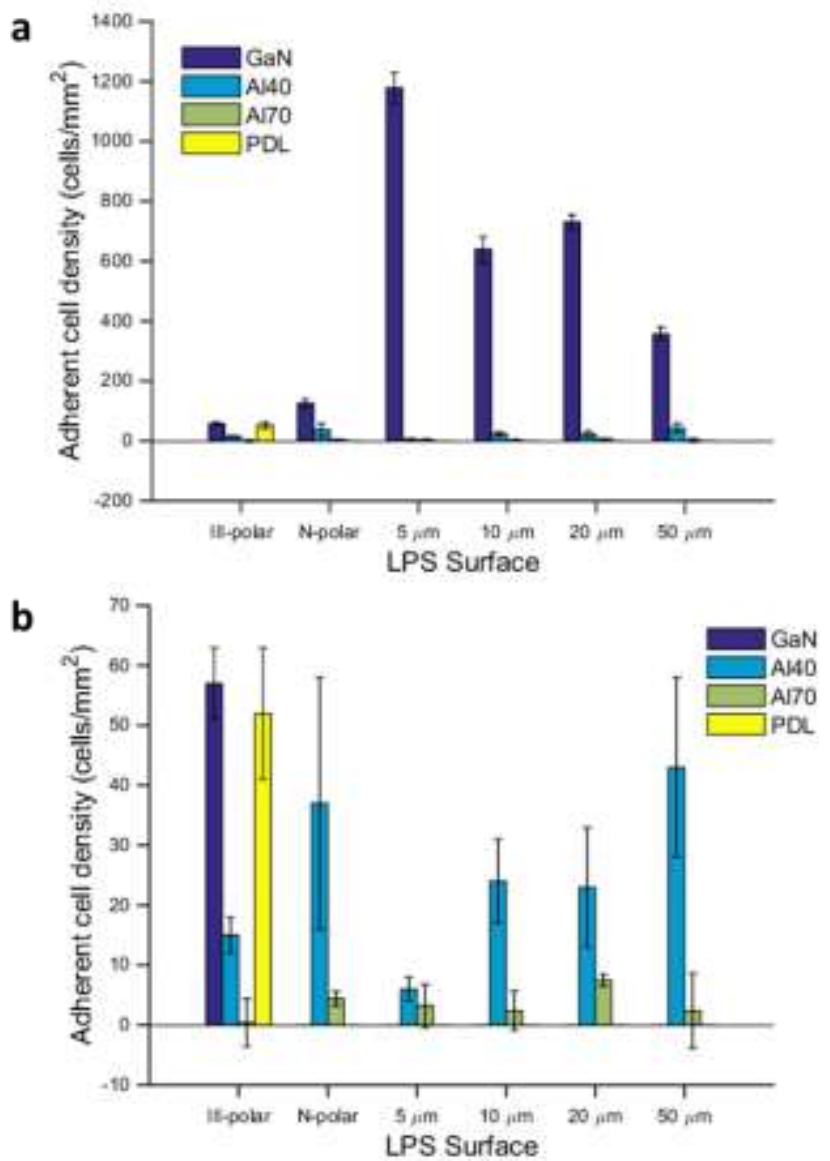

Figure 2 Summary of final (day 7) adherent cell density on LPS surfaces. (a) provides data for all surfaces, while (b) removes all but the Ga-polar region of $\mathrm{GaN}$ to emphasize both the reduced cell populations on the Al-containing wafers as well as the comparable density on Ga-polar GaN vs. PDL-coated sapphire. Error bars indicate \pm 1 standard deviation from the mean.

\section{PC12 Adhesion to the GaN LPS}

An AFM image of the $5 \mu \mathrm{m}$ pitch GaN wafer, provided in Figure 3 , provides a point of reference on the aforementioned 'step height difference' occurring at the interface between the two polarities. Full assessment of the nanoscale topography indicates that the height of the Ga-polar region is approximately 500-700 $\mathrm{nm}$ taller than the $\mathrm{N}$-polar regions. In addition to this periodic microscale pattern, there is an order of magnitude increase in roughness in the $\mathrm{N}$-polar region vs. the Ga-polar region (70 $\mathrm{nm}$ and $7 \mathrm{~nm}$ RMS, respectively.) While more subtle polarity-based interactions may be occurring at the cellmaterial interface to drive the observed changes in cell adhesion, surface topography has a well-documented impact on cellular adhesion and growth ${ }^{22}$. As such, this initial investigation of the cell response will largely emphasize these variations in topography.

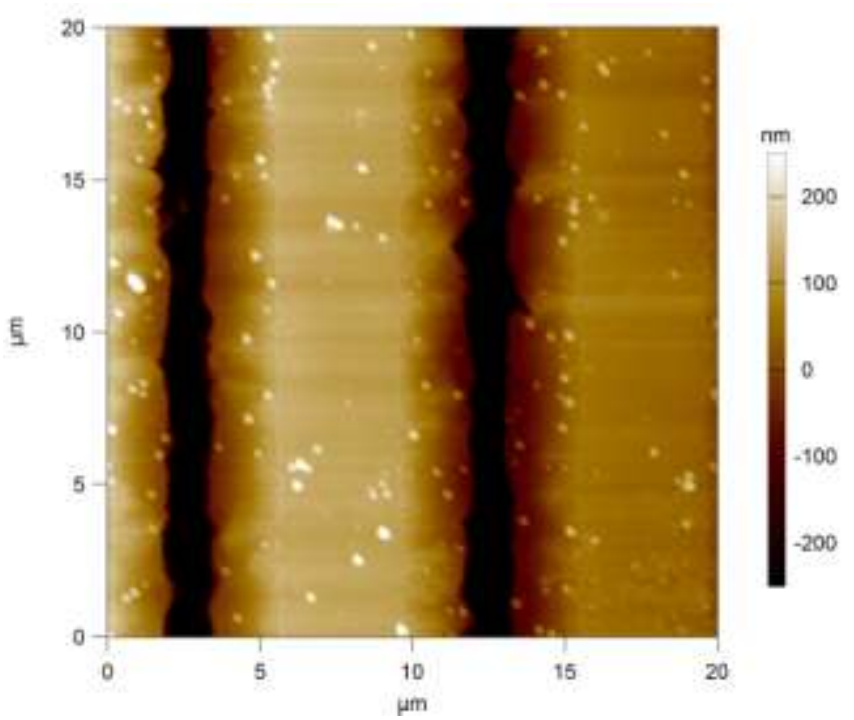

Figure 3 Atomic force micrography $\left(20 \times 20 \mu \mathrm{m}^{2}\right)$ of the GaN LPS, 5 $\mu \mathrm{m}$ region. Flat, light-colored regions are the Ga-polar material (vertical stripes, 5-10 and $15-20 \mu \mathrm{m}$ on the $\mathrm{x}$-axis.) These regions are both elevated with respect to and significantly less rough than the interstitial N-polar regions.

While cell populations on the Al-containing samples are significantly more limited, the number of adherent cells on the GaN LPS regions is adequate to draw larger scale observations of cellular behaviour - chiefly a potential relationship between cell size and LPS width that influences adherent cell density. PC12 cells have a diameter $\sim 13 \mu \mathrm{m}$ when grown on extra cellular matrix substrates. Given the topographic variation both in the increased roughness of $\mathrm{N}$-polar regions as well as the step height between III-polar and N-polar regions - across the surface, a GaN LPS width less than the diameter of the individual PC12 cells suggests that all cells are exposed to more diverse topographic features upon interaction with the surface. By contrast, if the GaN LPS features are larger than the cells (e.g. $50 \mu \mathrm{m}$ spacing, ) cells are less likely to come into contact with either a III/N-polarity boundary step or a rough $\mathrm{N}$-polar region. As such, we suggest that the increase in cell adhesion to the GaN LPS regions over the Ga-polar and N-polar surfaces is due to the change in nanoscale topography associated with the introduction of the polarity interface steps, and the greater 
density of these steps drives an increase in cell adhesion on the $5 \mu \mathrm{m}$ GaN LPS. On the two GaN LPS of comparable size to the average PC12 cell diameter (10 and $20 \mu \mathrm{m}$,$) a similar$ adherent cell density is observed, and again a reduction as $\mathrm{GaN}$ LPS width increases to $50 \mu \mathrm{m}$.

\section{Confirmation of Cell Adhesion to GaN vs. poly-D-lysine}

Poly-D-lysine (PDL) is used as a surface coating on cultureware for cells to improve attachment and neurotypic differentiation. Collagen coating is used for a similar reason; by introducing a positive charge to the culture surface, the affinity of neurotypic cells (e.g. PC12 cells) for adhering to that surface is improved. As reported by Chen and Young ${ }^{3}$ on cerebellar granule neuron growth on semiconductor materials, cells cultured on Si are generally less healthy and have a higher mortality rate than those cultured on PDL-coated polystyrene. By contrast, cells cultured on GaN adhered with higher density, formed a larger number of neuritic interconnections and synaptic junctions, and generally demonstrated healthier characteristic behaviours. ${ }^{3}$ To provide a greater degree of similarity in culture conditions, rather than coating the conventional polystyrene culture well with PDL, we coated a 2" sapphire wafer (the substrate wafers used for III-N material growth) and proceeded with the imaging experiment. Similar to the past study we found that initial cell attachment was comparable between the Ga-polar region of a GaN wafer and the PDL-coated sapphire. Moreover, adherent cell density in both regions was significantly less than that observed on the 10 $\mu \mathrm{m}$ GaN LPS (Fig. 4).

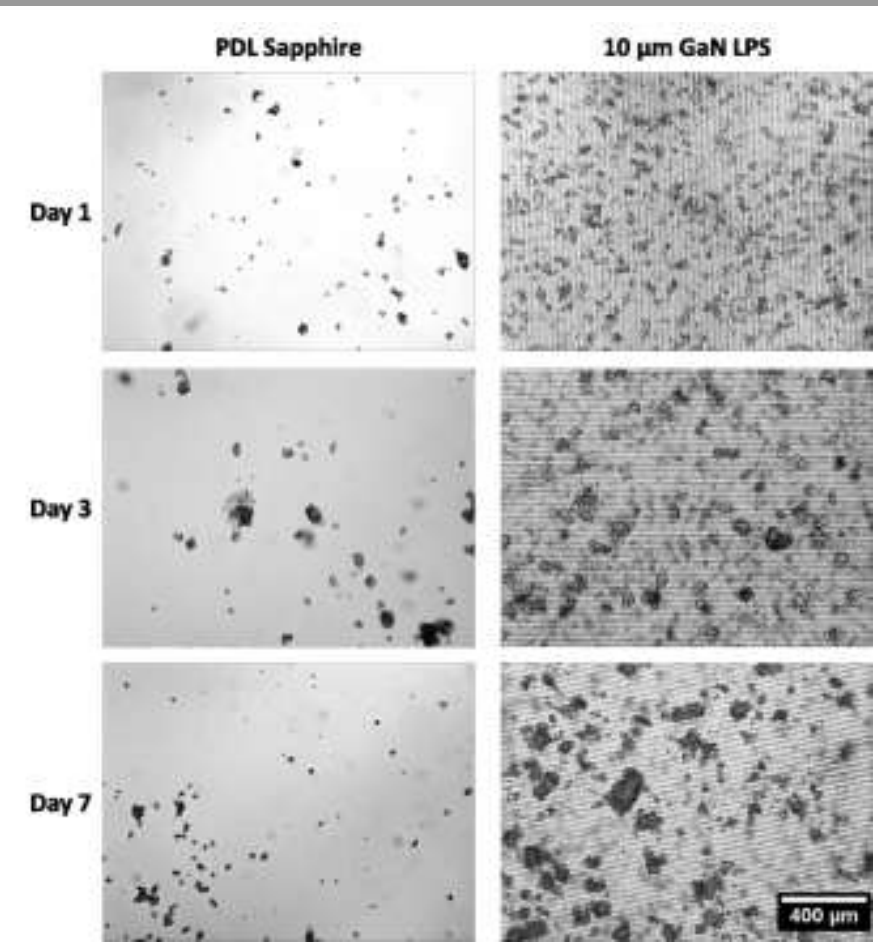

Figure 4 PC12 cells on PDL-coated sapphire vs. $10 \mu \mathrm{m}$ LPS GaN. A greater number of total cells and a higher density of the characteristics PC12 cell clusters are observed on the $10 \mu \mathrm{m}$ LPS at all time points. On the $10 \mu \mathrm{m}$ GaN LPS sample, out-of-focus debris is an artefact of the back-polishing to enable optical transparency. $400 \mu \mathrm{m}$ scale bar applies to all images.

\section{Cell Adhesion to Al- vs. Ga-rich Samples}

PC12 cells form aggregate clusters in culture, however, in data from both time-lapse imaging and longer time-point analyses, large (>5 cell) clusters tend to detach more readily from the surface. This is in part due to the nature of the clusters; only a small subset of the cluster cells are positioned such that they could form adhesions on the surface, and a smaller subset of that population successfully forms said adhesions. The presence of a bulk cluster without multiple adherent cells thus presents a large body more readily dislodged by the fluid shear associated with motion (either as the microscope stage shifts through imaging positions or as media is replaced.) As shown in Figure 5, a significantly higher number of multi-cell clusters are visible on the $\mathrm{GaN}$ wafer. Given the overall increase in adherent cell density on GaN, the longevity of cell clusters may be due to a higher incidence of multiple cells form a given cluster affiliating with the surface. As suggested by Podolska et al, increasing the compositional fraction of $\mathrm{Al}$ in an $\mathrm{AlGaN}$ substrate is known to reduce the confluence of human embryonic kidney cells following their initial attachment period. ${ }^{5}$ Our results suggest this holds true for neurotypic PC12 cells, as well. Al can form many oxide species in solution and the toxicity may be dependent on the form of $\mathrm{Al}$. Understanding the role of different oxides on toxicity is extremely important but a subject to a separate study being currently carried out in our laboratory. If this trend in confluence extends to multiple cells from a given cluster forming adhesions to the surface, the integrity of the clustersurface connection would be stronger. The increase in clusters on the GaN surface through time supports this concept; greater cluster-surface connectivity would provide a stronger connection more resistant to the shear stress induced by fluid motion.

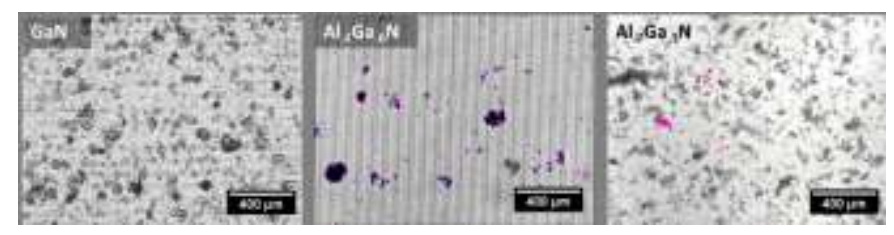

Figure 5 PC12 cells on the $10 \mu \mathrm{m}$ LPS. Images are from day 3 following NGF exposure. There is a significant reduction in adherent cells as the percentage of $\mathrm{Al}$ increases from 0 to 40 to 70 (the $100 \% \mathrm{Al}$ sample had no enduring attached cells.) Color contrast has been added to the cells in the AlGaN images to emphasize the sparse distribution. Scale bar $400 \mu \mathrm{m}$.

\section{Conclusions}

The growth and development of differentiating PC12 cells on a grating of alternating material polarity verified the sensitivity of PC12 cells to the composition and change in crystal polarization of their underlying growth substrate. This is indicative of the underlying phenomena driving surfacebiosystem interactions at the nanoscale; through both the 
surface topographic fluctuation as well as more nuanced changes associated with the local polarity of the crystalline material lattice, the chemical and physical nanostructure of the material surface does influence subsequent cellular interactions. Additionally, the apparent toxicity of AlN to neurotypic cell lines suggests that, a GaN capping layer may promote chemical stability and prevent potentially cytotoxic material interactions for future device construction intended for long-term neural cell exposure. Ongoing work to verify the chemical stability of the LPS samples as a function of $\mathrm{Al}$ :Ga composition will contribute to this understanding, providing a more comprehensive picture of the potential function of GaN/AIN/AlGaN-based materials in biomedical applications.

\section{Acknowledgements}

We thank ARO under W911NF-14-1-0664 and W911NF04-D-0003 for support of this work. We also thank NSF for partial financial support of this work under DMR-1312582 and DMR-1108071.

\section{Notes and references}

a UNC/NCSU Joint Department of Biomedical Engineering. North Carolina State University. Raleigh, NC.

${ }^{b}$ Department of Materials Science and Engineering. North Carolina State University. Raleigh, NC.

$c$ Department of Molecular Biomedical Sciences and Center for Comparative Medicine and Translational Research, College of Veterinary Medicine. North Carolina State University. Raleigh, NC.

1. Li, J.; Han, Q.; Zhang, Y.; Zhang, W.; Dong, M.; Besenbacher, F.; Yang, R.; Wang, C. Acs Applied Materials \& Interfaces 2013, 5, (19), 9816-9822.

2. Foster, C. M.; Collazo, R.; Sitar, Z.; Ivanisevic, A. Langmuir 2013, 29, (1), 216-220.

3. Chen, C. R.; Young, T. H. Biomaterials 2008, 29, (11), 1573-1582.

4. Jewett, S. A.; Makowski, M. S.; Andrews, B.; Manfra, M. J.; Ivanisevic, A. Acta Biomaterialia 2012, 8, (2), 728-733.

5. Podolska, A.; Tham, S.; Hart, R. D.; Seeber, R. M.; Kocan, M.; Kocan, M.; Mishra, U. K.; Pfleger, K. D. G.; Parish, G.; Nener, B. D. Sensors and Actuators B-Chemical 2012, 169, 401-406.

6. Ren, F.; Pearton, S. J. physica status solidi (c) 2012, 9, (2), 393-398.

7. Lopez-Gejo, J.; Arranz, A.; Navarro, A.; Palacio, C.; Munoz, E.; Orellana, G. Journal of the American Chemical Society 2010, 132, (6), 1746-+.

8. Lopez-Gejo, J.; Navarro-Tobar, A.; Arranz, A.; Palacio, C.; Munoz, E.; Orellana, G. Acs Applied Materials \& Interfaces 2011, 3, (10), 38463854 .

9. Ivanisevic, A.; Yeh, J. Y.; Mawst, L.; Kuech, T. F.; Ellis, A. B. Nature 2001, 409, (6819), 476-476.

10. Simpson, G. J.; Westerbuhr, S. G.; Rowlen, K. L. Analytical Chemistry 2000, 72, (5), 887-898.

11. Collazo, R.; Mita, S.; Rice, A.; Dalmau, R. F.; Sitar, Z. Applied Physics Letters 2007, 91, (21).

12. Collazo, R.; Mita, S.; Rice, A.; Dalmau, R.; Wellenius, P.; Muth, J.; Sitar, Z., Fabrication of a $\mathrm{GaN}$ p/n lateral polarity junction by polar doping selectivity. In Physica Status Solidi C - Current Topics in Solid State Physics, Vol 5, No 6, Palacios, T.; Jena, D., Eds. Wiley-V C H Verlag Gmbh: Weinheim, 2008; Vol. 5, pp 1977-1979.

13. Kirste, R.; Mita, S.; Hussey, L.; Hoffmann, M. P.; Guo, W.; Bryan, I.; Bryan, Z.; Tweedie, J.; Xie, J. Q.; Gerhold, M.; Collazo, R.; Sitar, Z. Applied Physics Letters 2013, 102, (18).

14. Kirste, R.; Mita, S.; Hoffmann, M. P.; Hussey, L.; Guo, W.; Bryan, I.; Bryan, Z.; Tweedie, J.; Gerhold, M.; Hoffmann, A.; Collazo, R.; Sitar, Z. Physica Status Solidi C: Current Topics in Solid State Physics, Vol 11, No 2 2014, 11, (2), 261-264.

15. Cordier, Y. physica status solidi (a) 2015, n/a-n/a.

16. Bain, L. E.; Ivanisevic, A. Small (Weinheim an der Bergstrasse, Germany) 2015, 11, (7).

17. Hu, W.; Yim, E. K. F.; Reano, R. M.; Leong, K. W.; Pang, S. W. Journal of Vacuum Science \& Technology B 2005, 23, (6), 2984-2989.

18. Foley, J. D.; Grunwald, E. W.; Nealey, P. F.; Murphy, C. J. Biomaterials 2005, 26, (17), 3639-3644.

19. Su, W. T.; Liao, Y. F.; Wu, T. W.; Wang, B. J.; Shih, Y. Y. Journal of Biomedical Materials Research Part A 2013, 101, (1), 185-194.

20. Hoffmann, M. P.; Kirste, R.; Mita, S.; Guo, W.; Tweedie, J.; Bobea, M.; Bryan, I.; Bryan, Z.; Gerhold, M.; Collazo, R.; Sitar, Z. physica status solidi (a) 2014, n/a-n/a.

21. Hoffmann, M. P.; Gerhold, M.; Kirste, R.; Rice, A.; Akouala, C. R.; Xie, J. Q. Q.; Mita, S.; Collazo, R.; Sitar, Z. Quantum Sensing and Nanophotonic Devices $X$ 2013, 8631.

22. Curtis, A.; Wilkinson, C. Biomaterials 1997, 18, (24), 1573-1583. 
